Research Paper

\title{
Combination therapy of human umbilical cord mesenchymal stem cells and FTY720 attenuates acute lung injury induced by lipopolysaccharide in a murine model
}

\author{
Zili Zhang ${ }^{1,2,4, *}$, Wenfei Li $^{3, *}$, Zhizhi Heng ${ }^{1}$, Jing Zheng ${ }^{1}$, Puyuan Li $^{1}$, Xin Yuan ${ }^{1}$, \\ Wenkai Niu ${ }^{1}$, Changqing Bai ${ }^{1}$ and Huiying Liu ${ }^{1}$ \\ ${ }^{1}$ Department of Respiratory and Critical Care Diseases, 307th Hospital of PLA, Beijing 100071, China \\ ${ }^{2}$ Anhui Medical University, Hefei 230032, China \\ ${ }^{3}$ School of Aerospace Medicine, Fourth Military Medical University, Xi'an 710032, China \\ ${ }^{4}$ Beijing Geriatric Hospital, Beijing 100095, China \\ *These authors have contributed equally to this work \\ Correspondence to: Changqing Bai, email: baicq307@163.com \\ Huiying Liv, email: huiyingliv1982@126.com \\ Keywords: acute lung injury, lipopolysaccharide, hUC-MSCS, FTY720, Sphingosine- 1-phosphate \\ Received: June 01, $2017 \quad$ Accepted: July 26, $2017 \quad$ Published: August 24, 2017 \\ Copyright: Zhang et al. This is an open-access article distributed under the terms of the Creative Commons Attribution License 3.0 \\ (CC BY 3.0), which permits unrestricted use, distribution, and reproduction in any medium, provided the original author and source \\ are credited.
}

\section{ABSTRACT}

ALI/ARDS remain the main reason of morbidity and mortality in the critically ill. Studies have indicated that human umbilical cord mesenchymal stem cells (hUCMSCs) can be useful in the treatment of ALI/ARDS. Sphingosine-1-phosphate (S1P) and its analog FTY720 significantly reduce lipopolysaccharide (LPS)-induced lung edema and inflammatory lung injury. This study aimed to assess the therapeutic effects of hUC-MSCs combined with FTY720 in an LPS-induced murine model of ALI. Eight-week-old female C57BL/6 mice were divided into a normal control group, an LPS group, an hUC-MSC group, an FTY720 group, and an hUC-MSCs+FTY720 group randomly. At 24 hours post injury, mice were administrated hUC-MSCs via the tail vein and/or intraperitoneally injected with FTY720. We assessed histopathology and histologic scores, lung wet/dry weight ratio, micro-CT scans, and total protein in the bronchoalveolar lavage fluid (BALF), as well as cytokines in the BALF at $48 \mathrm{~h}$ post injury. All treatment groups showed higher survival rates and attenuated lung injuries. The hUC-MSCs+FTY720 group yielded better results than hUC-MSCs or FTY720 alone. While the underlying mechanism requires further study, we anticipate that combination therapy of hUC-MSCs and FTY720 could be an effective strategy for ALI.

\section{INTRODUCTION}

Acute lung injury (ALI) and acute respiratory distress syndrome (ARDS) both can result in acute respiratory failure, and are remain the main reasons of death of critically ill patients [1]. These syndromes are induced by various clinical diseases and are characterized by serious intractable hypoxemia and increased inspired oxygen fraction [2]. Most treatments to improve hypoxemia in ARDS aim to improve aeration and ventilation [3]. Unfortunately, because of the short of effective therapy, the mortality is extremely high [4]. Lipopolysaccharide (LPS) is widely used for the establishment of ALI models. Recently, transplanting human mesenchymal stem cells or endothelial progenitor cells, was pointed out to alleviate ALI and decrease mortality induced by LPS [5-7]. In several researches $[8,9]$, MSCs are able to improve pathological damage, reduce inflammation, and even reduce mortality in a mouse model of ALI/ ARDS. These researches manifest that hUC - MSCs could be a new method of treatment for ALI/ ARDS [7]. 
Sphingosine-1-phosphate (S1P) is a kind of natural bioactive sphingolipid. It acts extracellularly via the $\mathrm{G}$ protein-coupled $\mathrm{S}_{1} \mathrm{P}_{1-5}$ as well as intracellularly on different aims, and mainly exists in tissues and plasma [10]. Some researches have indicated that S1P is a potent angiogenic factor. In preclinical animal models of ALI it can enhance lung endothelial cell integrity and is an inhibitor of vascular permeability and alveolar flooding [11-13]. In addition to S1P, S1P analogs, such as FTY720, provide a potential treatment in lung injury of murine models [14]. However, FTY720 decreased pulmonary function and increased the rate of dyspnea in a recent multiple clinical trial, not vice versa [15]. Thus, new mechanism findings are needed to lead to innovation strategies and treatments of lung injury [14].

In our previous study [16], we found that TNF- $\alpha$ expression was down-regulated more significantly if hUC-MSC was used in combination with S1P in a human pulmonary artery endothelial cells(HPAEC) model of acute injury. The combined effect mainly worked on S1PR2, S1PR3 and SphK2. When hUC-MSCs were combined with S1P, it also showed that the selectivity of S1P receptors was increased and the homeostatic control of S1P concentration was improved by regulating expressions of S1P metabolic enzymes [16].

ALI is an intricate pathophysiological process, and therapy targeted singly may not be the most effective. Therefore, we hypothesized that combination of transplantation of hUC-MSCs with FTY720 could reduce LPS-induced ALI in mice. In this study, we examined survival, histology, and pulmonary inflammation in ALI mice treated with hUC-MSCs, FTY720, or the combination of both.

\section{RESULTS}

\section{Combinatorial treatment superiorly attenuates LPS-induced lung injury}

CT scans of the mice lungs of all treatment groups at $48 \mathrm{~h}$ after LPS administration were generated. The results showed that compared with the LPS+PBS group, mice in LPS+hUC-MSCs, LPS+FTY720, and LPS+hUCMSCs+FTY720 groups showed clearly attenuated inflammation in the lungs after LPS challenge (Figure 1).

Left lung tissue sections were evaluated for edema, alveolar and interstitial inflammation, hemorrhage, and hyaline membrane formation. The sum of these scores was as a result of total lung injury score [18]. We selected each slide randomly and chosed high-power fields $(200 \times)$ to be analyzed, and the two investigators were blinded to the treatment groups (Figure 2). At $48 \mathrm{~h}$, the lung tissues of mice were found with inflammatory cell infiltration, hemorrhage, alveolar exudates, and edema. Also the injury scores were increased (Figure 3B). However, these pathological changes and injury scores were diminished in the LPS+hUC-MSCs, LPS+FTY720, and LPS+hUCMSCs+FTY720 groups as compared to the LPS+PBS group at this time point $(P<0.05)$. The effect was more significant in the LPS + hUC-MSCs + FTY720 group than in the other groups $(P<0.05)$. At day 7 , the histopathologic characteristics and injury scores were recovered to nearly normal in the control and treatment groups.

The wet/dry lung-weight ratio was determined to assess edema (Figure 3A). Lung edema was significantly alleviated at $48 \mathrm{~h}$ in the LPS+hUC-MSCs, LPS+FTY720, and LPS+hUC-MSCs+FTY720 groups as compared to the LPS+PBS group $(P<0.05)$, while there was no difference at 7 days.

\section{hUC-MSCs, FTY720, and combinatorial treatment groups had higher survival rate}

Four groups of mice ( $\mathrm{n}=15$ per group) were used for survival study. After the treatments, the mice recovered soon, and mortality was observed and recorded to $48 \mathrm{~h}$. hUC-MSCs, FTY720, and combinatorial treatment groups had higher survival rate compared with PBS group at 48 h. The survival rate of combination therapy group of FTY720 and hUC-MSCs was higher than other groups at $48 \mathrm{~h}$, although all the differences were not statistically significant (Figure 4).

\section{Combinatorial treatment superiorly attenuates vascular permeability and lung tissue inflammation}

Compared to the LPS + PBS group, total protein in BALF was significantly reduced in the LPS + hUC-MSCs, LPS+FTY720, and LPS+hUC-MSCs+FTY720 groups $(P<0.01)$ at $48 \mathrm{~h}$ and 7 days (Figure 5). The levels of inflammation marker MCP-1, IL-10, IL-12p70, TNF, IL6 , and IFN- $\gamma$ were measured in the BALF of mice at 48 $\mathrm{h}$ and 7 days after treatment for each group(Figure 6). TNF, IL-6, and MCP-1 were obviously lower in the three treatment groups than in the LPS+PBS group $(P<0.01)$. The decreases in TNF and MCP-1 were greater in the LPS+hUC-MSCs+FTY720 than in the two other groups $(P<0.01)$. However, no differences were founded in IL10 , IL-12p70 and IFN- $\gamma$ between the three treatments.

\section{DISCUSSION}

Previous studies have shown that hUC-MSCs home to injured lungs and differentiate into pulmonary epithelial cells and thus, can be regardred as a potential treatment for ALI [17]. Up to now, the mechanisms responsible for the treatment of MSCs in ALI are not entirely clear. Their capacity for pluripotent and secretion of multiple paracrine factors may be involved in these effects. Our previous study found that hUC-MSCs could impact on multiple $\mathrm{S} 1 \mathrm{P}$ related genes simultaneously as a potential therapy. 
The expression regulation result of related genes was not just the superposition of each other, but more obvious outcome, while hUC-MSCs were combined with S1P [16]. To further confirm the therapeutic effect of hUC-MSCs combined with SIP in the therapy of ALI, we used S1P analog FTY720 combined with hUC-MSCs to evaluate the therapeutic efficacy in LPS induced injury mice.

Our study showed that combination therapy of hUC-MSCs with FTY720 significantly attenuates ALI after LPS challenge as compared to each treatment alone. This suggests that FTY720 might help to improve the therapeutic effect of hUC-MSCs in ALI.

The primary target of this work was to investigate the therapeutic effects of combination therapy of hUCMSCs and FTY720 in ALI induced by LPS in mice. CT scans, lung histology, and injury scores suggested that the effects of the combination therapy were greater than those of hUC-MSCs or FTY720 alone. The major finding of this study was that combination therapy significantly improved the extent of lung injury, which was assessed quantitatively by the use of lung injury scores as described previously [18]. Increased capillary permeability in lung is considered to be a critical factor that protein-rich edema fluid infiltrate into the alveolar space [19]. Many researches have showed that MSCs and FTY720 treatment significantly reduce the lung wet/dry weight ratio and the protein in BALF [8, 20]. Consistently, our study indicated that lung edema and total protein in the BALF were obviously lowered after treatment for each group, with the combination therapy showing superior effect. ALI is an uncontrollable lung inflammation caused by a great quantity of inflammatory cells and cytokines [9]. The pro-inflammatory mediators TNF, MCP-1, and IL-6 were increased, which is evidence of acute systemic inflammatory response caused by LPS.
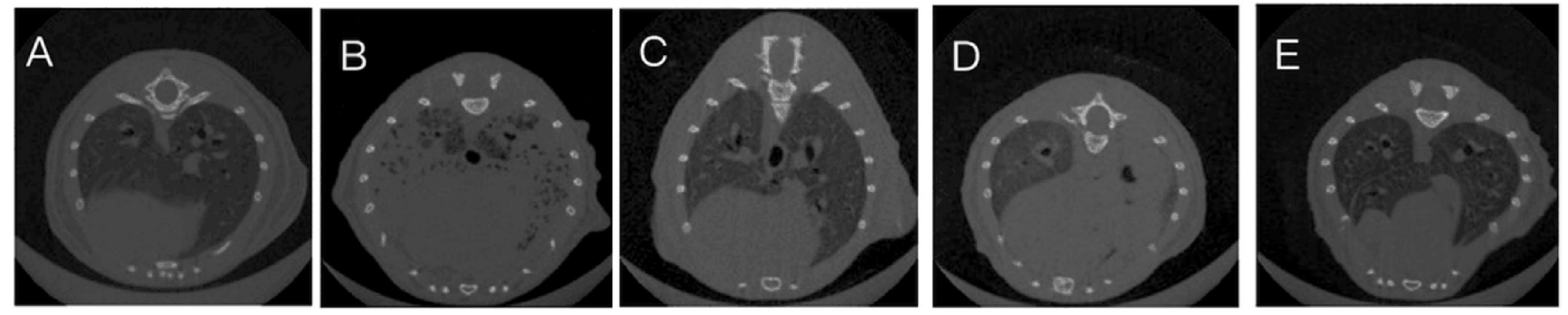

Figure 1: CT scans of lung tissues at $48 \mathrm{~h}$ after LPS challenge. (A) Control, (B) LPS+PBS, (C) LPS+hUC-MSCs, (D) LPS+FTY720, (E) LPS+ hUC-MSCs+ FTY720.

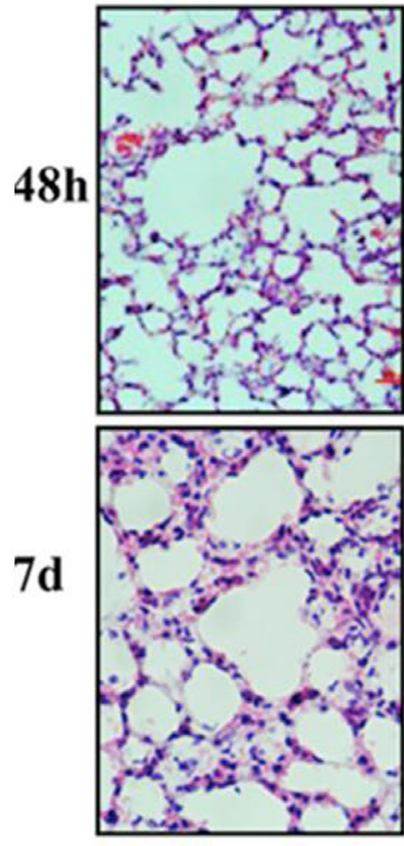

Control
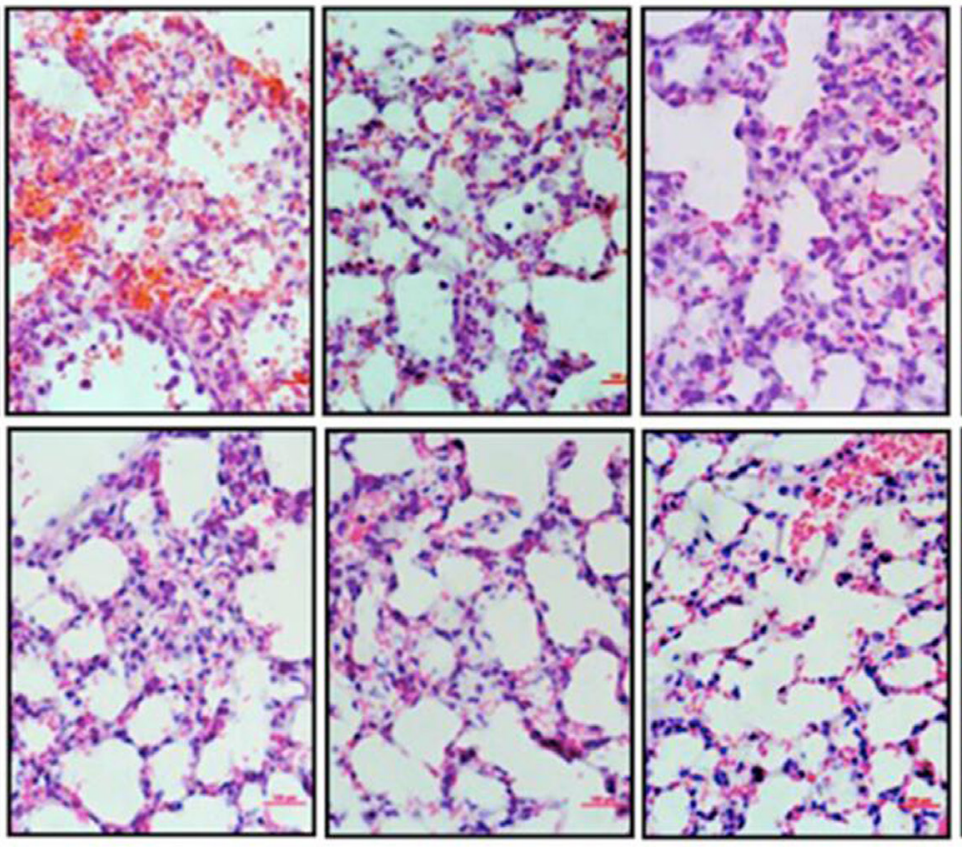

LPS+PBS

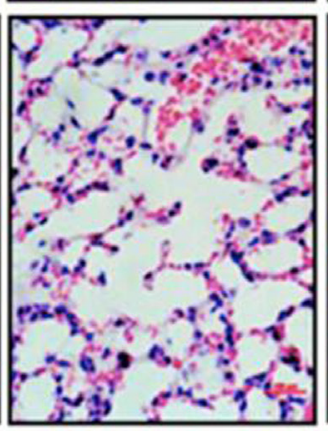

LPS+hUC-MSCs

LPS+FTY720

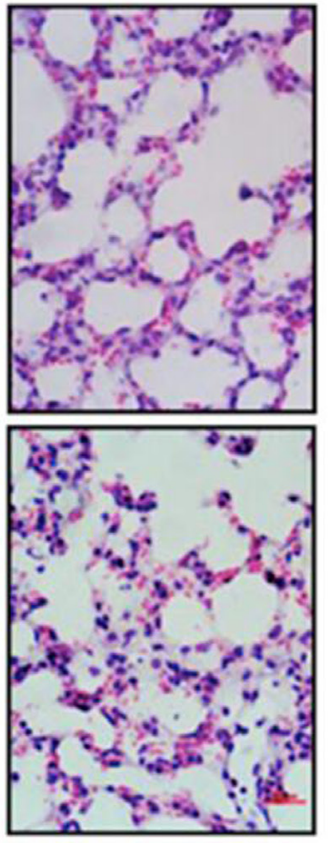

LPS+hUC-MSCs + FTY720

Figure 2: Histopathological images of lung tissue sections at $48 \mathrm{~h}$ and 7 days after LPS challenge (magnification, 200 $\times$ ). 


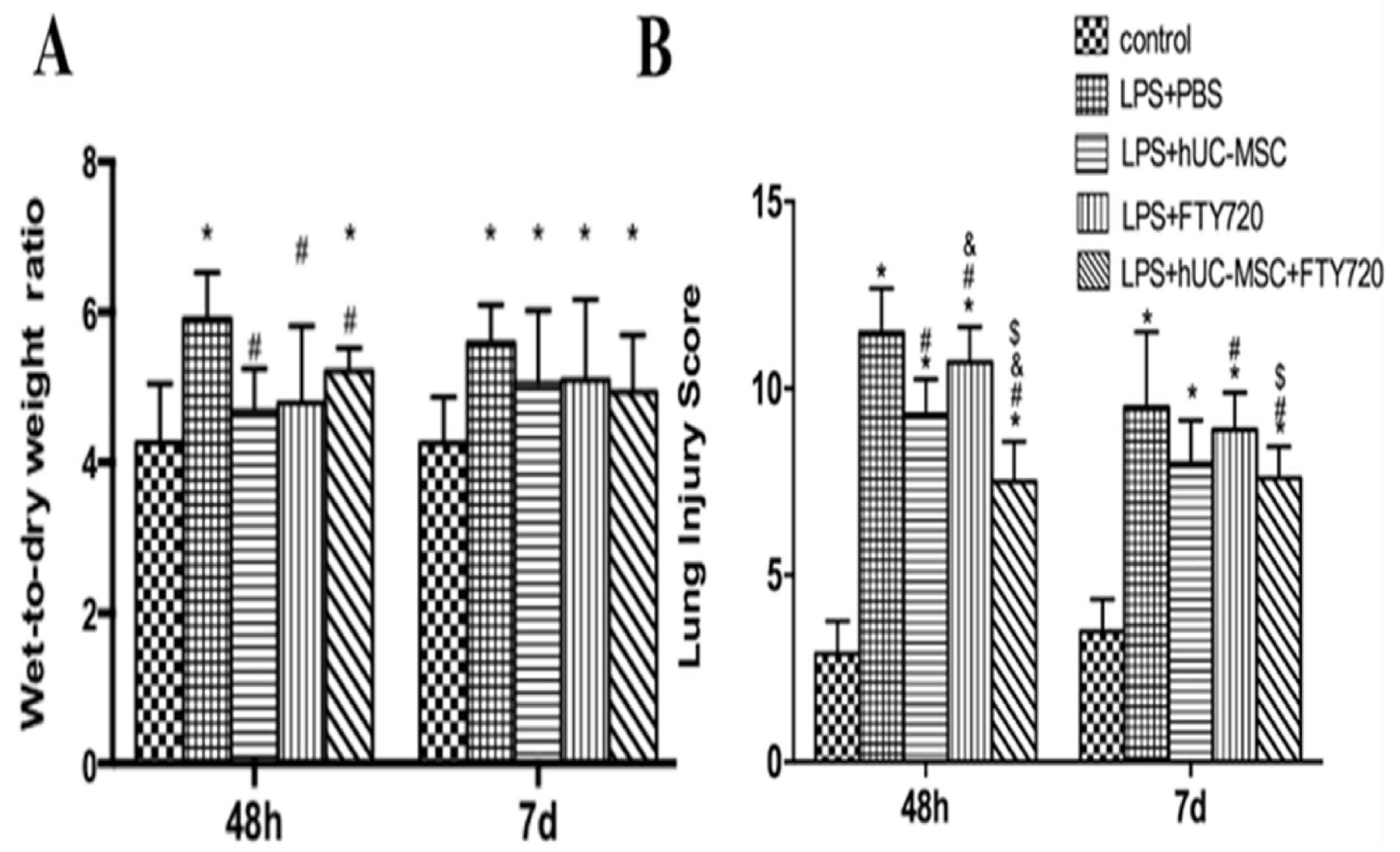

Figure 3: Quantification of lung edema and injury in LPS-challenged mice. (A) Lung edema was determined as the lung wet/ dry weight ratio. Results are shown for samples taken at $48 \mathrm{~h}$ and 7 days after LPS challenge. (B) Lung injury scores show an obvious decrease in the severity of lung injury in the LPS+hUC-MSCs and LPS+FTY720 groups, and especially, in the LPS+hUC-MSCs+FTY720 group. Data are represented by the mean $\pm \mathrm{SD}, \mathrm{n}=10$ at each time point for each group. ${ }^{*} P<0.05$ vs. control; ${ }^{\#} P<0.05$ vs. LPS $+\mathrm{PBS} ;{ }^{\&} P$ $<0.05$ vs. LPS+ hUC-MSCs; ${ }^{\$} P<0.05$ vs. LPS+FTY720.

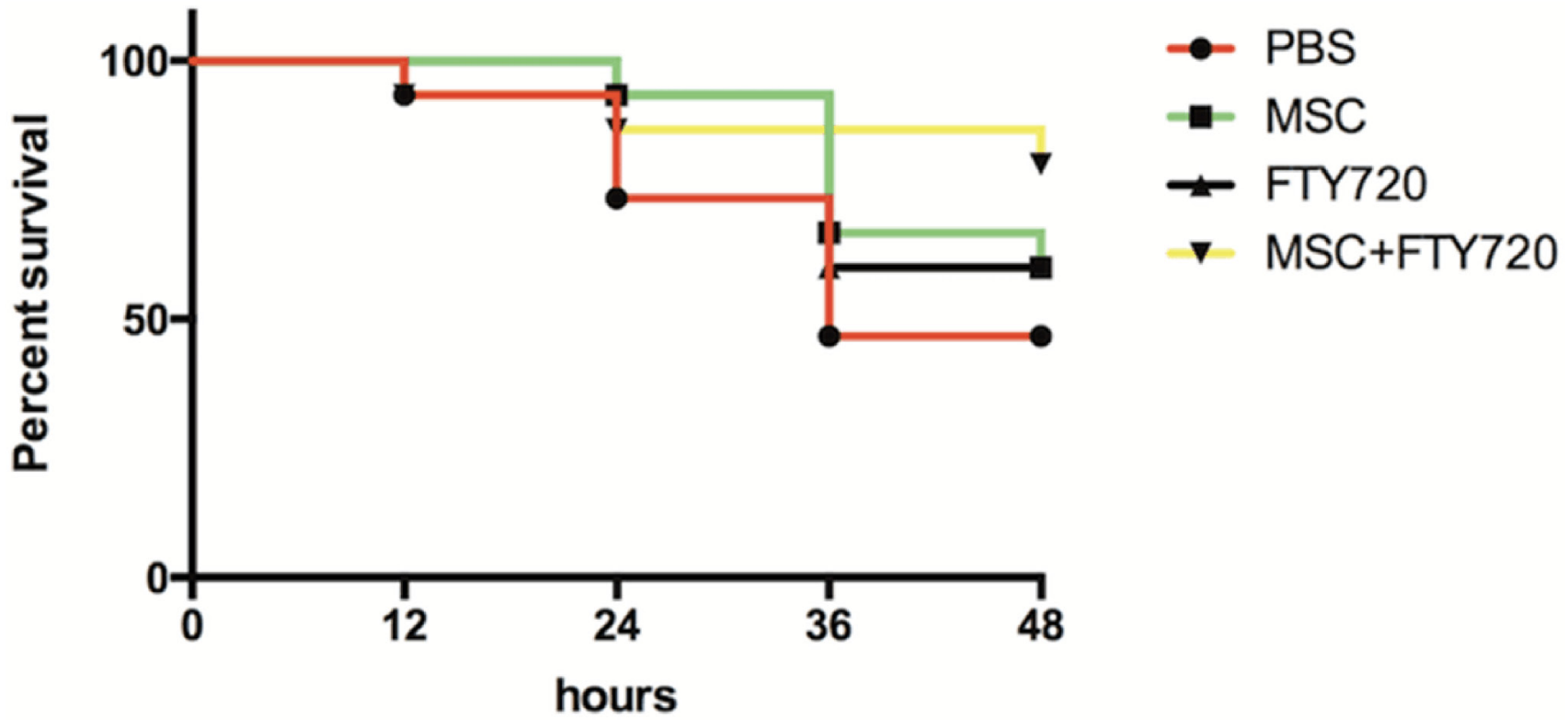

Figure 4: Mouse survival rates ( $\mathrm{n}=15$ for each group) at $48 \mathrm{~h}$ after LPS challenge using Kaplan-Meier analysis followed by a log-rank test. The survival of mice in the LPS+hUC-MSCs+FTY720 group was higher than that in other experimental groups, but there were not statistically significant in the differences $(P=0.07$, LPS+hUC-MSCs+FTY720 vs. LPS + PBS; $P=0.29$ LPS+hUC-MSCs+FTY720 vs. LPS+hUC-MSCs; $P=0.31$ LPS+ hUC-MSCs+FTY720 vs. LPS+FTY720). 


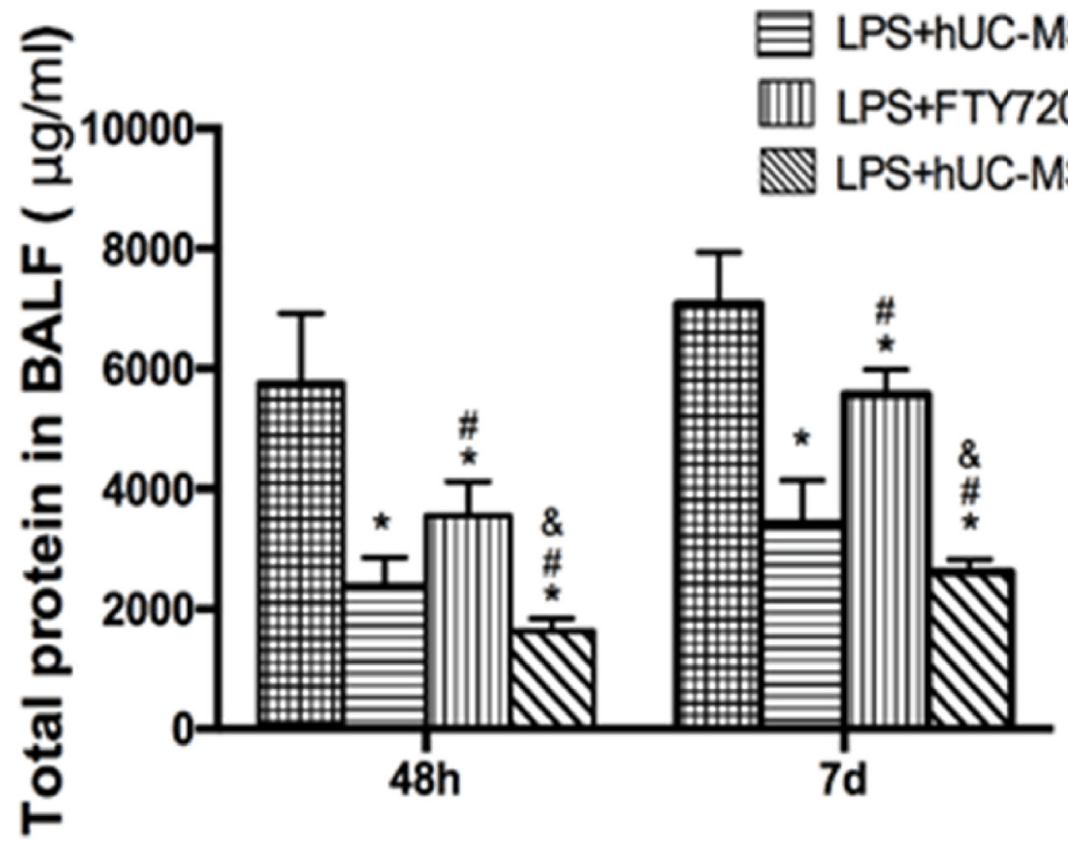

Figure 5: Total protein concentration in the BALF at $48 \mathrm{~h}$ and 7 days after LPS administration. Data are represented by the mean $\pm \mathrm{SD}\left(\mathrm{n}=10\right.$ at each time point for each group). ${ }^{*} P<0.01 \mathrm{vs}$. LPS $+\mathrm{PBS} ;{ }^{*} P<0.01 \mathrm{vs}$. LPS $+\mathrm{hUC}-\mathrm{MSCs} ;{ }^{\circledR} P<0.01 \mathrm{vs}$. LPS+FTY720.
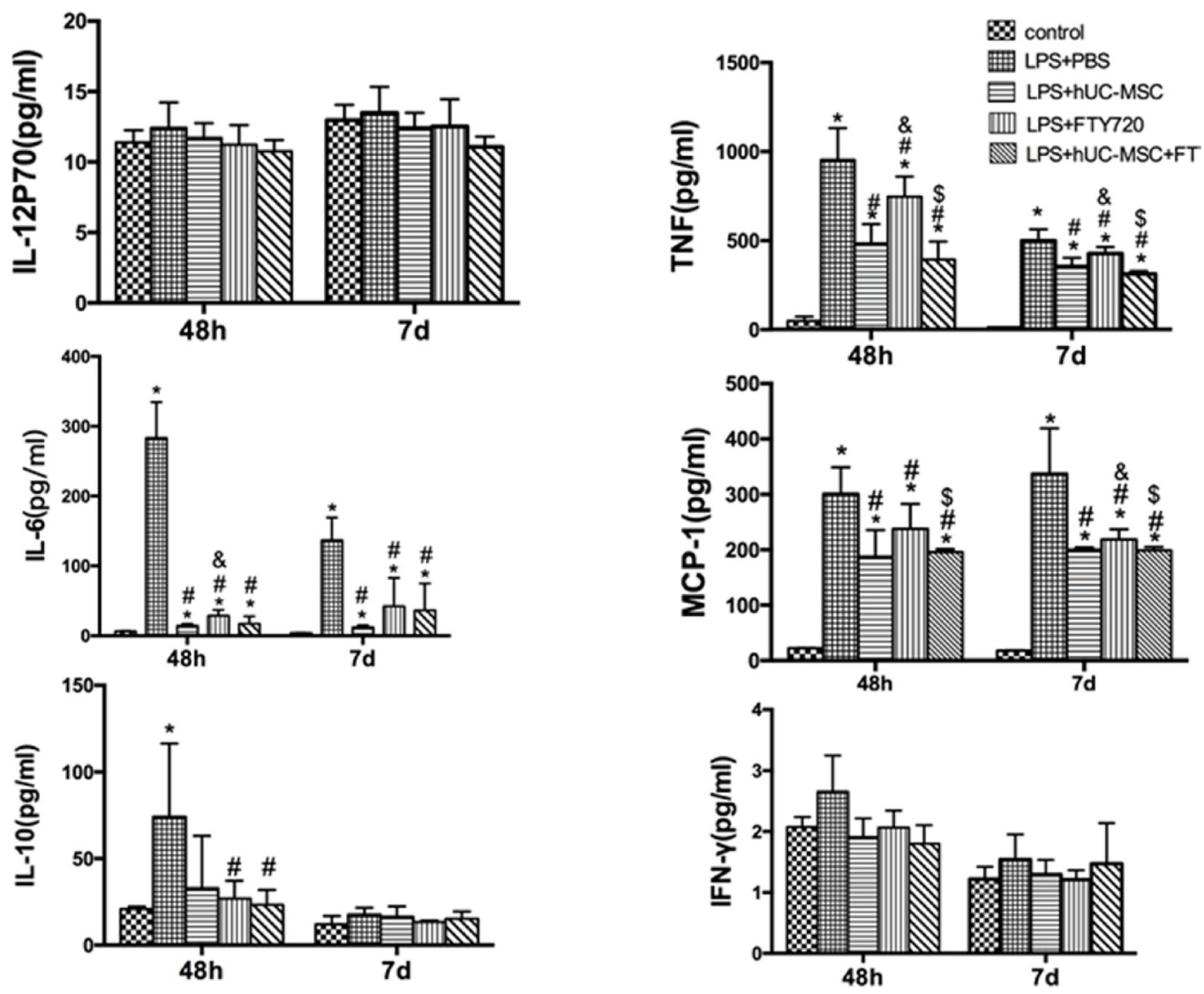

Figure 6: Inflammatory cytokine levels in the BALF at $48 \mathrm{~h}$ and 7 days after LPS administration. BALF was obtained for measuring these indicators using mouse inflammation kits. Data are represented by the mean $\pm \mathrm{SD}(\mathrm{n}=6$ at each time point for each group). ${ }^{*} P<0.01$ vs. control; ${ }^{\sharp} P<0.05$ vs. LPS+PBS; ${ }^{\circledR} P<0.05$ vs. LPS+hUC-MSCs; ${ }^{\$} P<0.05$ vs. LPS+FTY720. 
Earlier reports suggested that LPS challenge increases IL-6 secretion and pulmonary leakage [21]. The decrease in anti-inflammatory could be one of the key mechanisms of the treatment.

Some studies reaffirmed the conclusion that an antagonistic relationship existed between S1PR1 and S1PR2 in the vascular endothelium during tissue injury and disease [22]. In an acute injury model of HPAEC, it found that the expressions of S1PR1-3 all increased. However, the expression of S1PR1 was not obviously changed while the expressions of S1PR2 and S1PR3 decreased significantly when hUC-MSCs were given [16]. We speculate that if hUC-MSCs could up-regulate the $\mathrm{S} 1 \mathrm{P}$ receptors selectivity in vivo, so as to enhance the endothelial barrier function.

Clinical studies have indicated that no single target or drug can reverse the serious pathological damage caused by ARDS/ALI and achieve the expected treatment outcome rapidly. Combination therapy of FTY720 with hUC-MSCs may be a new approach to treatment for reducing the high mortality and morbidity caused by ALI in humans.

\section{MATERIALS AND METHODS}

\section{Animals}

8 to 12 weeks female C57BL/6 mice were purchased from Beijing HFK Bioscience Co. (Beijing, China). They were keeped in individual cages with free access to water and food (Laboratory Animal Center of the Academy of Military Medical Sciences, Beijing, China). All animal experiments conformed to NIH guidelines (Guide for the care and use of laboratory animals) (NIH Publication No. 85-23, revised 2011). We divided mice into 5 treatment groups randomly: a normal control (control) group, a PBS control (LPS+PBS) group, an hUC-MSC treatment (LPS+hUC-MSCs) group, an FTY720 treatment (LPS+FTY720) group, and an hUC-MSC plus FTY720 treatment (LPS+hUC-MSCs+FTY720) group. The mice were all euthanized finally.

\section{Establishment of the ALI mouse model}

To induce ALI, we anesthetized mice with pentobarbital sodium and then intratracheally instilled with Escherichia coli O55:B5 LPS (10 mg/kg body weight, $50 \mu \mathrm{l}$; Sigma, St. Louis, MO). Mice were then recovered again as before, and survival was noted. The mice were sacrificed for assessment after 48 hours or 7 days and samples were collected according to the different experiments.

\section{hUC-MSCs and FTY720 treatments}

Mice were given an injection of PBS or hUC-MSCs $\left(2 \times 10^{5}\right.$ cells $)$ in a $200-\mu l$ volume via the tail vein at $24 \mathrm{~h}$ after the LPS challenge. hUC-MSCs were obtained from the 307-IVY Translation Medicine Center (Laboratory of Oncology, Affiliated Hospital of Academy of Military Medical Sciences, Beijing, China). A single intraperitoneal injection of FTY720 reduces murine lung injury obviously and low concentrations of FTY720 (0.1 mg/kg) significantly reduce lung permeability $[11,23]$; therefore, mice in the LPS+FTY720 and LPS+hUC-MSCs+FTY720 groups were given an intraperitoneal injection of FTY720 (0.1 mg/kg, $200 \mu \mathrm{l}$; Sigma, St. Louis, MO) at 24h after LPS administration.

\section{Sample acquisition}

Lungs were harvested at $48 \mathrm{~h}$ and 7 days after LPS administration. Left lung lobes were fixed in $10 \%$ formalin, paraffin-embedded, and sectioned at $5-\mu \mathrm{m}$ thickness. BAL fluid (BALF) was collected at $48 \mathrm{~h}$ by flushing $1 \mathrm{ml}$ of ice-cold PBS back and forth three times through a tracheal cannula with a 20-gauge catheter to assess concentrations of protein and inflammatory factors as indexes of lung permeability (injury). BALF was centrifuged at $1,500 \times \mathrm{g}$ for $10 \mathrm{~min}$ at $4^{\circ} \mathrm{C}$ and stored at $-80^{\circ} \mathrm{C}$ until analysis.

\section{Histological analysis}

Lung tissue sections were stained with hematoxylin and eosin and mice were examined by micro-CT (PerkinElmer-Caliper LS, Co., Boston, MA) for lung morphology. A scoring system to grade the degree of lung injury was used, based on the following histologic features: edema, hyperemia and congestion, neutrophil margination, and tissue infiltration. Edema, alveolar and interstitial inflammation, hemorrhage, and hyaline membrane formation were each scored using a 0 to 4 point scale (no injury, 0 ; injury in $25 \%$ of the field, 1 ; injury in $50 \%, 2$; injury in $75 \%, 3$; and injury throughout the field, $4)$. The sum of these scores was as a result of total lung injury score [18].

\section{Lung wet/dry weight ratio measurement}

After the mice were sacrificed, right lungs were excised, blotted dry, and weighed. Lung dry weights were recorded after the samples were dried in an oven at $80^{\circ} \mathrm{C}$ for $12 \mathrm{~h}$. The lung wet/dry weight ratio was used to assess the degree of pulmonary edema.

\section{Total protein and inflammatory factors in the BAL}

Total protein concentration in the BALF was examined by a standard BCA kit (BD Biosciences, San Jose, CA). Inflammatory markers IL-12p70, IL-10, IL-6, TNF, MCP-1, and IFN- $\gamma$ in the BALF were examined by BD Cytometric Bead Array (CBA) mouse inflammation kit (BD Biosciences) per the manufacturer's instructions. 


\section{Statistical analysis}

The data are expressed as the mean \pm SD. KaplanMeier analysis followed by a log-rank test was used to analyse survival rates. Group means were compared using a $t$-test. All data were analyzed using SPSS version 17.0 and GraphPad Prism 5. A $P$-value $<0.05$ was considered statistically significant.

\section{Abbreviations}

ALI: acute lung injury. ARDS: acute respiratory distress syndrome. hUC-MSCs: human umbilical cord mesenchymal stem cells. S1P: Sphingosine-1phosphate. S1PR: Sphingosine-1-phosphate receptor. LPS: lipopolysaccharide. BALF: bronchoalveolar lavage fluid. IL: interleukin. TNF- $\alpha$ : tumor necrosis factor- $\alpha$. CT: computed tomography. PBS: phosphate buffer saline. MCP-1: monocyte chemotactic protein-1. IFN: interferon. CBA: cytometric bead array. HPAEC : human pulmonary artery endothelial cells.

\section{CONFLICTS OF INTEREST} interests.

The authors declare that they have no competing

\section{FUNDING}

This work was supported by research grants from the Beijing Natural Science Foundation (7164284); Innovation Science Research Foundation of 307th Hospital of PLA (FC-2014-01); Public Health Major Project: AWS15J007.

\section{REFERENCES}

1. Matthay MA, Ware LB. The acute respiratory distress syndrome. N Engl J Med. 2000; 342: 1334-1349. https:// doi.org/10.1056/NEJM200005043421806.

2. ARDS Definition Task Force, Ranieri VM, Rubenfeld GD, Thompson BT, Ferguson ND, Caldwell E, Fan E, Camporota L, Slutsky AS. Acute respiratory distress syndrome: the Berlin Definition. JAMA. 2012; 307: 25262533. https://doi.org/10.1001/jama.2012.5669.

3. Pelosi P, de Abreu MG. Acute respiratory distress syndrome: we can't miss regional lung perfusion! BMC Anesthesiol. 2015; 15: 35. https://doi.org/10.1186/s12871-015-0014-z.

4. Rubenfeld GD, Caldwell E, Peabody E, Weaver J, Martin DP, Neff M, Stern EJ, Hudson LD. Incidence and outcomes of acute lung injury. N Engl J Med. 2005; 353: 1685-1693. https://doi.org/10.1056/NEJMoa050333.

5. Kim ES, Yun SC, Choi SJ, Jin KK, Yoo HS, Ahn SY, Dong KS, Kim SY, Ye RP, Park WS. Intratracheal transplantation of human umbilical cord blood-derived mesenchymal stem cells attenuates Escherichia coli -induced acute lung injury in mice. Respir Res. 2011; 12: 1-11. https://doi. org/10.1186/1465-9921-12-108.

6. Cribbs SK, Martin GS. Stem cells in sepsis and acute lung injury. Am J Med Sci. 2011; 341: 325-332. https://doi. org/10.1097/MAJ.0b013e3181f30dee.

7. Lee JW, Gupta N, Serikov V, Matthay MA. Potential application of mesenchymal stem cells in acute lung injury. Expert Opin Biol Ther. 2009; 9: 1259-1270. https://doi. org/10.1517/14712590903213651.

8. Gupta N, Su X, Popov B, Lee JW, Serikov V, Matthay MA. Intrapulmonary delivery of bone marrow-derived mesenchymal stem cells improves survival and attenuates endotoxin-induced acute lung injury in mice. J Immunol. 2007; 179: 1855-1863. https://doi.org/10.4049/ jimmunol.179.3.1855.

9. Li J, Li D, Liu X, Tang S, Wei F. Human umbilical cord mesenchymal stem cells reduce systemic inflammation and attenuate LPS-induced acute lung injury in rats. J Inflamm (Lond). 2012; 9: 33. https://doi. org/10.1186/1476-9255-9-33.

10. Merrill AH Jr, Schmelz EM, Dillehay DL, Spiegel S, Shayman JA, Schroeder JJ, Riley RT, Voss KA, Wang E. Sphingolipids--the enigmatic lipid class: biochemistry, physiology, and pathophysiology. Toxicol Appl Pharmacol. 1997; 142: 208-225. https://doi.org/10.1006/taap.1996.8029.

11. Mcverry BJ, Peng X, Hassoun PM, Sammani S, Simon BA, Garcia JG. Sphingosine 1-phosphate reduces vascular leak in murine and canine models of acute lung injury. Am J Respir Crit Care Med. 2004; 170: 987-993. https://doi. org/10.1164/rccm.200405-684OC.

12. Peng X, Hassoun PM, Sammani S, McVerry BJ, Burne MJ, Rabb H, Pearse D, Tuder RM, Garcia JG. Protective effects of sphingosine 1-phosphate in murine endotoxininduced inflammatory lung injury. Am J Respir Crit Care Med. 2004; 169: 1245-1251. https://doi.org/10.1164/ rccm.200309-12580C.

13. Sanchez T, Estrada-Hernandez T, Paik JH, Wu MT, Venkataraman K, Brinkmann V, Claffey K, Hla T. Phosphorylation and action of the immunomodulator FTY720 inhibits vascular endothelial cell growth factorinduced vascular permeability. J Biol Chem. 2003; 278: 47281-47290. https://doi.org/10.1074/jbc.M306896200.

14. Natarajan V, Dudek SM, Jacobson JR, Morenovinasco L, Huang LS, Abassi T, Mathew B, Zhao Y, Wang L, Bittman R. Sphingosine-1-phosphate, FTY720, and Sphingosine-1phosphate receptors in the pathobiology of acute lung injury. Am J Respir Cell Mol Biol. 2013; 49: 6-17. https:// doi.org/10.1165/rcmb.2012-0411TR.

15. Kappos L, Antel J, Comi G, Montalban X, O'Connor P, Polman CH, Haas T, Korn AA, Karlsson G, Radue EW. Oral fingolimod (FTY720) for relapsing multiple sclerosis. N Engl J Med. 2006; 355: 1124-1140. https://doi.org/10.1056/ NEJMoa052643. 
16. Liu H, Zhang Z, Li P, Yuan X, Zheng J, Liu J, Bai C, Niu W. Regulation of S1P receptors and sphingosine kinases expression in acute pulmonary endothelial cell injury. PeerJ. 2016; 4: e2712. https://doi.org/10.7717/peerj.2712.

17. Cai S, Liu A, Chen S, He H, Chen Q, Xu J, Pan C, Yang Y, Guo F, Huang Y. Activation of Wnt $/ \beta$-catenin signalling promotes mesenchymal stem cells to repair injured alveolar epithelium induced by lipopolysaccharide in mice. Stem Cell Res Ther. 2015; 6: 65. https://doi.org/10.1186/ s13287-015-0060-y.

18. Mrozek JD, Smith KM, Bing DR, Meyers PA, Simonton SC, Connett JE, Mammel MC. Exogenous surfactant and partial liquid ventilation. Am J Respir Crit Care Med. 1997; 156: 1058-1065. https://doi.org/10.1164/ ajrccm.156.4.9610104.

19. Liu KD, Matthay MA. Advances in critical care for the nephrologist: acute lung injury/ARDS. Clin J Am Soc Nephrol. 2008; 3: 578-586. https://doi.org/10.2215/ CJN.01630407.

20. Camp SM, Bittman R, Chiang ET, Moreno-Vinasco L, Mirzapoiazova T, Sammani S, Lu X, Sun C, Harbeck M,
Roe M, Natarajan V, Garcia JG, Dudek SM. Synthetic analogs of FTY720 (2-amino-2-(2-(4-octylphenyl)ethyl)1,3-propanediol) differentially regulate pulmonary vascular permeability in vivo and in vitro. J Pharmacol Exp Ther. 2009; 331: 54-64. https://doi.org/10.1124/jpet.109.153544.

21. Schwab SR, Pereira JP, Matloubian M, Xu Y, Huang Y, Cyster JG. Lymphocyte sequestration through S1P lyase inhibition and disruption of S1P gradients. Science. 2005; 309: 1735-1739. https://doi.org/10.1126/science.1113640.

22. Blaho VA, Hla T. An update on the biology of sphingosine 1-phosphate receptors. J Lipid Res. 2014; 55: 1596-1608. https://doi.org/10.1194/jlr.R046300.

23. Müller HC, Hocke AC, Hellwig K, Gutbier B, Peters H, Schönrock SM, Tschernig T, Schmiedl A, Hippenstiel S, N'Guessan PD, Rosseau S, Suttorp N, Witzenrath M. The Sphingosine-1 phosphate receptor agonist FTY720 dose dependently affected endothelial integrity in vitro and aggravated ventilator-induced lung injury in mice. Pulm Pharmacol Ther. 2011; 24: 377-385. https://doi. org/10.1016/j.pupt.2011.01.017. 